Biol. Cybern. 61, 327-345 (1989)

\title{
Processing of Figure and Background Motion in the Visual System of the Fly
}

\author{
W. Reichardt, M. Egelhaaf, and Ai-ke Guo* \\ Max-Planck-Institut für biologische Kybernetik, Spemannstrasse 38, D-7400 Tübingen, Federal Republic of Germany
}

\begin{abstract}
The visual system of the fly is able to extract different types of global retinal motion patterns as may be induced on the eyes during different flight maneuvers and to use this information to control visual orientation. The mechanisms underlying these tasks were analyzed by a combination of quantitative behavioral experiments on tethered flying flies (Musca domestica) and model simulations using different conditions of oscillatory large-field motion and relative motion of different segments of the stimulus pattern. Only torque responses about the vertical axis of the animal were determined. The stimulus patterns consisted of random dot textures ("Julesz patterns") which could be moved either horizontally or vertically. Horizontal rotatory large-field motion leads to compensatory optomotor turning responses, which under natural conditions would tend to stabilize the retinal image. The response amplitude depends on the oscillation frequency: It is much larger at low oscillation frequencies than at high ones. When an object and its background move relative to each other, the object may, in principle, be discriminated and then induce turning responses of the fly towards the object. However, whether the object is distinguished by the fly depends not only on the phase relationship between object and background motion but also on the oscillation frequency. At all phase relations tested, the object is detected only at high oscillation frequencies. For the patterns used here, the turning responses are only affected by motion along the horizontal axis of the eye. No influences caused by vertical motion could be detected. The experimental data can be explained best by assuming two parallel control systems with different temporal and spatial integration properties: The
\end{abstract}

* On leave of absence from the Institute of Biophysics, Academia Sinica, Beijing, People's Republic of China
$L F$-system which is most sensitive to coherent rotatory large-field motion and mediates compensatory optomotor responses mainly at low oscillation frequencies. In contrast, the $S F$-system is tuned to small-field and relative motion and thus specialized to discriminate a moving object from its background; it mediates turning responses towards objects mainly at high oscillation frequencies. The principal organization of the neural networks underlying these control systems could be derived from the characteristic features of the responses to the different stimulus conditions. The input to the model circuits responsible for the characteristic sensitivity of the SF-system to small-field and relative motion is provided by retinotopic arrays of local movement detectors. The movement detectors are integrated by a large-field element, the output cell of the network. The synapses between the detectors and the output cells have nonlinear transmission characteristics. Another type of large-field elements ("pool cells") which respond to motion in front of both eyes and have characteristic direction selectivities are assumed to interact with the local movement detector channels by inhibitory synapses of the shunting type, before the movement detectors are integrated by the output cells. The properties of the LF-system can be accounted for by similar model circuits which, however, differ with respect to the transmission characteristic of the synapses between the movement detectors and the output cell; moreover, their pool cells are only monocular. This type of network, however, is not necessary to account for the functional properties of the LF-system. Instead, intrinsic properties of single neurons may be sufficient. Computer simulations of the postulated mechanisms of the SF- and LF-system reveal that these can account for the specific features of the behavioral responses under quite different conditions of coherent large-field motion and relative motion of different pattern segments. 


\section{Introduction}

Motion information is critically important for many biological systems. The nervous system is confronted with motion information in two principally different, though not mutually exclusive, situations: when an object moves in front of the eyes but also when the animal itself moves through a stable environment. The resulting retinal image displacements depend in a characteristic way on the trajectory of the moving object, the animal's own direction and speed, as well as on the three-dimensional structure of the visual environment. If evaluated properly by the nervous system, this information can be used in visual orientation. For instance, deviations of the animal from its course inevitably lead to coherent displacements of the retinal image of the entire visual scene, which contain a strong rotational component. When this component is extracted from the retinal motion pattern, it can be used to mediate compensatory optomotor responses of the animal which may lead to a stabilization of its course. In contrast, when the animal passes nearby objects located in front of a more distant background, the retinal images of these objects and their background move relative to each other leading to discontinuities in the retinal motion field. Relative motion thus may indicate the existence of nearby stationary or moving objects. This information can be used to discriminate objects from their background and thus enable the animal to respond to them, for instance, by orientational turning responses. Since these global retinal motion patterns are particularly conspicious in fast moving animals and, especially, in flying ones, their visual systems can be expected to be highly adapted to evaluate motion information.

This is one reason that the fly proved to be a good model system for analyzing the neuronal computations by which global retinal motion patterns are extracted and transformed into different components of visual orientation behavior. In the fly visual system, the computations underlying the evaluation of two types of global motion patterns, compensatory optomotor turning reactions mediated by coherent rotatory large-field motion and orientational turning responses induced by small-field and relative motion, have been investigated in great detail by both behavioral and electrophysiological techniques as well as by theoretical analysis. In the last years, our efforts mainly concentrated on the mechanisms underlying the extraction of relative motion, originally at the algorithmic level, but later also at the level of neural networks (Virsik and Reichardt 1976; Reichardt and Poggio 1979; Poggio et al. 1981; Reichardt et al. 1983; Egelhaaf 1985a-c).

Retinal image displacements along the horizontal axis of the eye could be shown to play an important role in mediating both compensatory optomotor turning responses and turning responses towards objects. These motion components are sensed by twodimensional retinotopic arrays of local movement detectors (for review, see Reichardt 1987). There is good experimental evidence that both behavioral tasks are controlled, although with a varying sensitivity, by local movement detectors from allover the eye. This means that the visual control systems under consideration here have a large number of parallel input channels provided by the photoreceptors and their subsequent local movement detectors, but eventually only a single output variable, i.e. yaw torque. As a consequence, the input channels have to be spatially pooled somewhere between the local movement detectors and the final motor output. This spatial integration is not linear. In house- and blowflies, it takes place separately in two pathways which are specifically tuned to different types of global retinal motion patterns (for review, see Egelhaaf et al. 1988). The large-field ( $L F-$ ) system is most sensitive to coherent rotatory large-field motion, whereas the small-field ( $\mathrm{SF}_{-}$) system is most sensitive to the motion of small moving objects as well as to relative motion of the objects and their background. Both control systems were initially characterized at the behavioral level. Only later have specific classes of output cells of the optic lobes been attributed to the proposed spatially integrating elements in these two pathways. This has been mainly done by correlating specific functional properties of the behavioral responses and visual interneurons (Reichardt et al. 1983; Wehrhahn and Hausen 1980; Hausen 1982a, b, 1984; Egelhaaf 1985a-c, 1987; Hausen and Egelhaaf 1989). In addition specific evidence has also been derived from microsurgical lesioning (Hausen and Wehrhahn 1983, 1989) or laser ablation (Geiger and Nässel 1982) of specific neuronal structures as well as on the basis of behavioral mutants in Drosophila (Heisenberg and Wolf 1984; Bausenwein et al. 1986). Two functional classes of output elements of the optic lobes were shown in this way to be particularly important. While the HS-cells are most sensitive to large-field rotatory image displacements and, therefore, are believed to be an integral part of the LF-system (Hausen 1982a, b), the FD-cells respond preferentially to small moving targets as well as to relative motion and, therefore, were interpreted as being constituent elements of the SF-system (Egelhaaf 1985b, c). The LF- and the SFsystem were proposed to aquire their characteristic spatial integration properties by interactions with other types of large-field elements ("pool cells") which represent specific aspects of large-field motion and serve as a kind of reference signal with respect to the single movement detector channels (Poggio et al. 1981; Reichardt et al. 1983; Egelhaaf 1985a, c). 
Since the turning responses are jointly mediated by at least two parallel control systems, the specific characteristics of the behavioral responses cannot easily be related to the functional properties of any particular neuronal element. This was only possible when it was realized that the LF- and the SF-field system have different dynamical properties (Egelhaaf 1987). While the LF-system controls yaw torque strongest at low oscillation frequencies, the SF-system contributes to yaw torque mainly at high oscillation frequencies. This allows to analyze the functional properties of both control systems relatively independently even at the behavioral level and to relate their functional characteristics to the properties of the HS- and FD-cells in a much more specific way than was possible before. This will be the objective of the present study.

By means of behavioral experiments we try to constrain the directional selectivities and binocular interactions of the input circuitries of both the LF- and SF-system. The turning responses of the fly to coherent large-field motion and relative motion of an object and its background will be analyzed. In particular, it will be studied under what conditions of relative motion the object can be discriminated from its background. Then we analyze whether the input circuits of the LF- and the SF-system are only affected by motion along the horizontal axis of the eye or also by vertical motion. Then we further constrain, on the basis of experiments with four independently moving pattern segments, the input organization of the networks with respect to the horizontal axis of the eye. This input organization will finally be formulated in terms of neuronal model circuits.

\section{Materials and Methods}

In the experiments reported here mechano-electrical transducers were used which enable us to measure behavioral responses of fixed flying flies. The transducer senses the flight torque of a test fly around its vertical axes as generated by the forces of the wings (e.g. Fermi and Reichardt 1963, Götz 1964). The experiments were carried out on female wild type Musca domestica from our laboratory stock. Flies were prepared as originally described by Fermi and Reichardt (1963). The head of the test animals was fixed to the thorax with a mixture of wax and colophonium in order to exclude head movements. Under these conditions, a resting pattern represents a stabilized retinal image for the test fly. The preparation was carried out under light carbon dioxide anesthesia. A triangular piece of cardboard was fixed to the wax just above the frontal part of the thorax to mount a testfly to the compensator. The ocelli were covered with the same mixture of colophonium and wax. The flies were given
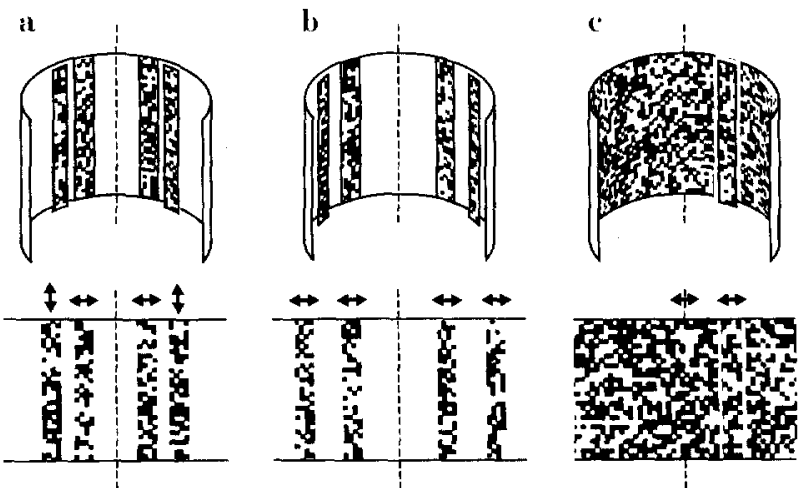

Fig. 1a-c. Schematic representation of the different stimulus conditions used in the experiments. In all experiments, the test fly was suspended from a torque meter (not shown) in the center of a cylindrical stimulus panorama. In the upper diagrams the cylinder is opened to allow the stimulus pattern to be seen from behind. The stimulus patterns consisted of a random texture (pixel size: $3^{\circ} \times 3^{\circ}$ ). In the bottom diagrams the different stimulus patterns are illustrated in a more stylized way. In the set-up shown in a four vertical stripes of $12^{\circ}$ width were used. They were located at mean angular horizontal positions of $\psi \pm 40^{\circ}$ and $\psi \pm 20^{\circ}$, respectively. The two frontal stripes were oscillated horizontally, the lateral ones vertically. In the set-up shown in $b$, four vertical stripes were used. However, they were only oscillated horizontally about the mean horizontal positions of $\psi= \pm 60^{\circ}$ and $\psi= \pm 30^{\circ}$. In c only a single vertical stripe at an angular position of $\psi=+30^{\circ}$ as seen from the fly's frontal midline was oscillated in front of an equally textured background. The oscillation amplitude amounted always to $\pm 3^{\circ}$ in a and to $\pm 6^{\circ}$ in $b$ and $c$

at least one day before an experiment to recover from the preparation.

Three different kinds of stimulus conditions were used:

(1) Four different stripes, at radial distances of $36 \mathrm{~mm}$ and $35 \mathrm{~mm}$ from the testfly, were oscillated (Fig. 1a). The angular widths of the stripes (measured from the center of a testfly) was $12^{\circ}$. The two frontal stripes positioned at $\psi= \pm 20^{\circ}$ - were oscillated together horizontally, whereas the two lateral stripes - positioned at $\psi= \pm 40^{\circ}$ - were oscillated vertically $(\psi$ denotes the horizontal angular position with respect to the frontal midline of the animal). The oscillation amplitudes of the three stripes amounted to $\pm 3^{\circ}$. The frontal and lateral stripes were oscillated with different relative phases. Phase $0^{\circ}$ is defined as follows: When the frontal stripes move to the right (seen from the testfly) the lateral stripes move upward. The measurements were carried out with relative phases of $0^{\circ}, 90^{\circ}$, $180^{\circ}$, and $270^{\circ}$ and at three different frequencies ( 2.44 , $1.22,0.244 \mathrm{~Hz}$ ).

(2) Four different stripes with the same angular width $\left(12^{\circ}\right)$ positioned at radial distances of $36 \mathrm{~mm}$ and $35 \mathrm{~mm}$ from the testfly were oscillated horizontally (Fig. 1b). 
The oscillation amplitudes of the four stripes amounted to $\pm 6^{\circ}$. The two frontal stripes - positioned at $\psi= \pm 30^{\circ}$ - were oscillated together, whereas the two lateral stripes - positioned at $\psi= \pm 60^{\circ}$ - were always moved with a $180^{\circ}$ phase shift with respect to each other. The relative phase between the lateral stripe on the right side and the two frontal stripes amounted to $0^{\circ}, 90^{\circ}, 180^{\circ}$, and $270^{\circ}$. The experiments were carried out at four different frequencies $(2.44,1.22,0.244$, $0.122 \mathrm{~Hz}$ ).

(3) In this set-up figure-ground discrimination could be tested. A testfly was confronted with a textured background cylinder and an equally textured stripe ("figure") (Fig. 1c). Both figure and background could be oscillated together or independently. The oscillation amplitudes amounted to $\pm 6^{\circ}$. The width of the stripe was $12^{\circ}$. Figure-ground discrimination was tested for four relative phases $\left(0^{\circ}, 90^{\circ}, 180^{\circ}, 270^{\circ}\right)$ and for four different frequencies $(2.44,1.22,0.244$, $0.122 \mathrm{~Hz}$ ). In all set-ups the stripes as well as the background in set-up (3) consisted of random dot textures (pixel size $3^{\circ} \times 3^{\circ}$ ) with a contrast of $78 \%$ and a mean luminance of about $700 \mathrm{~cd} \cdot \mathrm{m}^{-2}$.

The experiments carried out with set-ups (2) and (3) were simulated with a Hewlett-Packard computer (300 Series). The model simulations were based on a model consisting of $121 \times 121$ local movement detectors, a LF- and SF-system which integrate the detector outputs parallel to the equator of the eyes. The final motor output of the model is controlled by the summated output signals of the LF- and SF-system via a direct pathway and a channel which computes their running average. In the experiments the pixel size of a random dot pattern or of random dot stripes amounted to $3^{\circ} \times 3^{\circ}$. Since an individual stripe has a width of $12^{\circ}$ it is made up of 4 pixels in a row. In the computer simulations we made use of the following approximations: (1) The network is assumed to be spatially isotropic. Under these conditions the stripe in a figureground experiment may be located separately from the ground neglecting the overlap of both patterns. The response produced by a random dot pattern or by a random dot stripe was derived from the response of an individual pixel multiplied by the size of the particular pattern. This is justified by the finding made in computer experiments that little influence is observed on the response whether the pixel is oscillated with large or with small amplitudes. (3) The simulations were carried out with an individual pixel covering an array of $33 \times 33$ movement detectors and oscillating with a small amplitude. (4) The continuous temporal oscillations of the four stripes or of figure and ground were approximated by 64 individual vectors. The delay in an individual detector was frequency independent and amounted to 1/64 of the temporal period.

\section{Results}

\section{Torque Responses Induced by Coherent Large-Field Motion and Relative Motion}

Figure-ground discrimination by relative motion in the visual system of the fly was first demonstrated in a series of closed loop experiments (Virsik and Reichardt 1974, 1976). In these experiments a fly was suspended from a torque compensator and controlled by its own torque response the angular velocity of stimulus patterns. Relative motion between object and ground enables a fly to fixate and to track the object, even if the structure of both patterns is statistically equivalent. This finding is corroborated by open-loop experiments where the fly cannot influence the motion of its optical environment (Reichardt and Poggio 1979; Reichardt et al. 1983).

Contrary to the earlier experiments (Reichardt and Poggio 1979; Reichardt et al. 1983) which were restricted to either time averaged behavioral responses or to only one oscillation frequency $(2.5 \mathrm{~Hz})$, the experiments presented here were carried out at different frequencies $(2.44,1.22,0.244,0.122 \mathrm{~Hz})$. The experiments were done with set-up (3) as described in Materials and Methods and shown in Fig. 1c.

Figure 2 shows the outcome of these experiments. At the beginning of each experiment, figure and ground oscillate synchronously, i.e. with a phase shift of $\phi=0^{\circ}$. Then the relative phase is switched to either $\phi=0^{\circ}, 90^{\circ}$, $180^{\circ}$ or $270^{\circ}$ (indicated by the arrow-heads at the bottom of the diagrams). During synchronous oscillation, the yaw torque oscillates about the straightahead direction irrespective of the oscillation frequency. This suggests that the fly responds with optomotor turning reactions which, in free flight, would minimize the relative velocity between stimulus and eyes and thus stabilize the flight course. The response amplitudes, however, are much smaller at high than at low oscillation frequencies (see Egelhaaf 1987).

After three cycles of synchronous oscillation, figure and ground start moving relative to each other. The figure may then, at least in principle, be distinguished from its background. However, whether it is distinguished by the fly depends not only on the phase relationship between figure and ground, but also on the oscillation frequency of the pattern. At high frequencies and all phase shifts between figure and ground motion tested here, the time course and the mean response amplitudes change considerably. Since the shape of the response profiles are characteristic fingerprints of the different phase relations, they can be used to constrain the possible model circuits likely to be responsible for the visually induced turning responses. Moreover, for all phase relations a mean torque response is generated towards the position of 

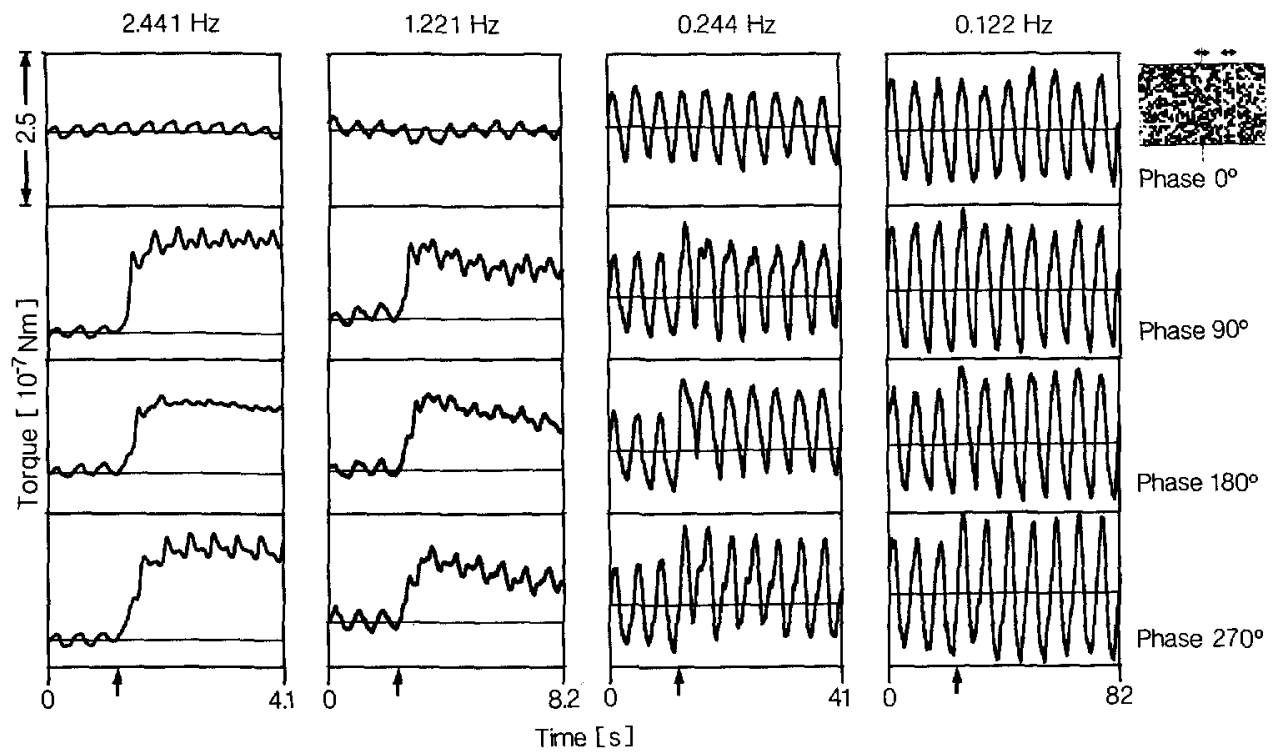

Fig. 2. Torque responses to coherent rotatory large-field motion and relative motion of the background and the vertical stripe (stimulus set-up (3); see inset) to different oscillation frequencies (indicated above the different columns) and phase relations (indicated at the right of each horizontal row). During synchronous oscillation (phase $0^{\circ}$ ) the flies show only oscillatory torque responses about a mean zero response level (indicated in each diagram by the thin horizontal line). Only the response amplitude increases with decreasing oscillation frequency. When the phase between the oscillation of the background and the stripe is switched to relative motion (indicated by the arrow-head at the bottom of the diagrams), the torque responses at high oscillation frequencies change considerably. Irrespective of the phase relationship, the mean responses are positive. The time course of the responses, however, are a characteristic fingerprint of the particular phase relationship. The data are averages taken from 10 different flies each stimulated 10 times with the same stimulation program

the figure which, in free animals, would bring the figure in front of the eyes. This indicates that the fly has detected the figure and is trying to fixate it. It should be noted that this result is in agreement with findings on Drosophila (Bülthoff 1981) but, at least partly, in contrast to earlier studies on the large flies Musca and Calliphora (Reichardt and Poggio 1979; Reichardt et al. 1983). Here, the fly did not show any significant indication of figure-ground discrimination during counter-phase oscillation of figure and ground. It should be mentioned, however, that our present sample of flies showed some variability in this respect: Some flies did not show any pronounced change in their mean response amplitude when the phase was switched to $\phi=180^{\circ}$; however, when averaged over the entire population of flies, pronounced mean positive responses are obvious (Fig. 2).

At low oscillation frequencies, neither the mean amplitude nor the time course of the responses to relative motion with all phase shifts tested here differ much from those elicited by synchronous motion of figure and ground. Hence, the fly does not respond to the figure in any obvious way. It can thus be concluded that for equally textured patterns relative motion is necessary, but not sufficient for figure-ground discrimination. The figure is only discriminated from the ground at high oscillation frequencies.

\section{Is Spatial Integration Parallel to the Eyes' Equator Independent of Integration Orthogonal to it?}

In order to determine how the outputs of the movement detectors control the flight motor, it is essential to understand the organization of the spatial integration of the local movement detectors. For the sake of argument, let us assume a two-dimensional array of orthogonally oriented movement detectors. The horizontally aligned detectors control the optomotor responses around the vertical body axis, whereas the vertical detectors control the turning responses about the long body axis. This simple organization would be more complicated if pool-cells integrating over all horizontally and vertically oriented detectors were assumed to influence via a separate forward channel the direct channels before these converge to control the behavioral responses (see Sect. 4).

To test this possibility by behavioral experiments the set-up (1) as described in Materials and Methods (Fig. 1a) was used. The pair of frontal stripes as well as the pair of lateral stripes always oscillated in synchrony. The two lateral stripes, however, were oscillated with varying their phase relations with respect to the frontal stripes. In order to determine the phase relations between the two pairs of stripes one has to introduce a convention: If the frontal stripes are 


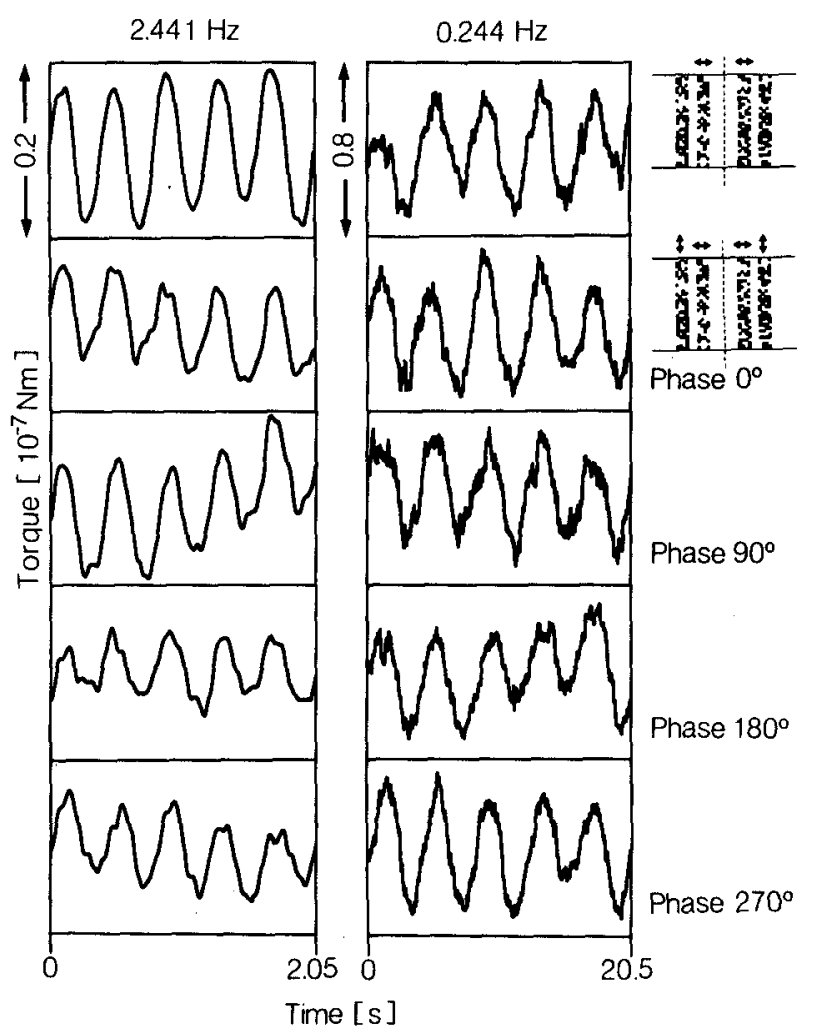

Fig. 3. Torque responses to the motion of a pair of horizontally and a pair of vertically moving stripes (stimulus set-up (1); see inset) obtained at two oscillation frequencies (indicated above the columns) and different relative phases between the two pairs of stripes (indicated at the right; the convention for defining the different phase relations is given in the text). In the upper row only the two frontal stripes are oscillated (see inset). At both high and low oscillation frequencies, the torque responses are not affected in any obvious way by the vertically moving stripes. The data are averages obtained from 10 different flies each stimulated 10 times with the same stimulation program

moved to the right (as seen from the testfly) and the lateral stripes are moved upward, the relative phase between the two pairs is defined as being zero. The oscillation frequency amounted to either $2.44,1.22$ or $0.244 \mathrm{~Hz}$. At each of these frequencies five different experiments were carried out: Only the pair of frontal stripes was oscillated, or both pairs were oscillated with different relative phases of $0^{\circ}, 90^{\circ}, 180^{\circ}$ or $270^{\circ}$.

The experimental results for 2.44 and $0.244 \mathrm{~Hz}$ are shown in Fig. 3. The results for $1.22 \mathrm{~Hz}$ are virtually identical to the results for the other two frequencies. They indicate that periodic torque responses are generated under all stimulus conditions. The amplitudes of the responses increase with decreasing oscillation frequencies. The important point, however, is that the responses measured at each frequency for different relative phase relations do not differ from each other in any obvious way. Hence, the vertically moving stripes have neither a direct nor an indirect influence on the responses elicited by the horizontally oscillating stripes. These observations suggest that there are no interactions between internal representations of both horizontally and vertically directed motion defectors.

\section{Spatial Integration Properties Along the Horizontal Axis of the Eye}

The organziation of the input circuitry of both the LFand the SF-system was further constrained in experiments done with set-up (2) as described in Materials and Methods (see Fig. 1b) with four different oscillation frequencies $(2.44 \mathrm{~Hz}, 1.22,0.244$, and $0.122 \mathrm{~Hz}$ ). At the beginning of all experiments only the two frontal stripes were oscillated synchronously in phase (Fig. 4). Irrespective of the oscillation frequency, the flies try to follow the stimulus panorama by generating oscillatory yaw torque responses of the same frequency as the stimulus motion. However, as already described for the results of Sects. 1 and 2 the response amplitude strongly depends on the oscillation frequency. It is much larger at low oscillation frequencies than at high ones. Since the optic stimuli oscillate symmetrically with respect to the dorso-frontal symmetry axis of the animal, the resulting yaw torque responses can be assumed to be symmetrical about the zero line (indicated by the thin line in the different diagrams of Fig. 4). This zero response level will be used as line of reference while evaluating the responses under the other stimulus conditions.

After these cycles of synchronous motion of the frontal pair of stripes, the stimulus conditions change and the lateral stripes start oscillating. The frontal stripes continue oscillating in phase. In contrast, the lateral stripes always oscillate in counterphase. While the phase relations within each pair of stripes (the frontal and the lateral pair of stripes, respectively) remains fixed, the phase between the pairs was varied in different experiments. When related to the motion of the frontal pair of stripes, the phase of the lateral stripe in front of the right eye amounted to $0^{\circ}, 90^{\circ}, 180^{\circ}$ or $270^{\circ}$ (This implies that the phase of the left lateral stripe was then $180^{\circ}, 270^{\circ}, 0^{\circ}$ or $90^{\circ}$, respectively). In the following we always refer to the phase between the pair of frontal stripes and the right lateral stripe.

One important feature of the experimental data is obvious at first glance when comparing the response profiles obtained at the different oscillation frequencies. At low oscillation frequencies neither the time course of the responses to the different phase relations nor their mean value differ much from those elicited by synchronous motion of the frontal stripes alone. The 

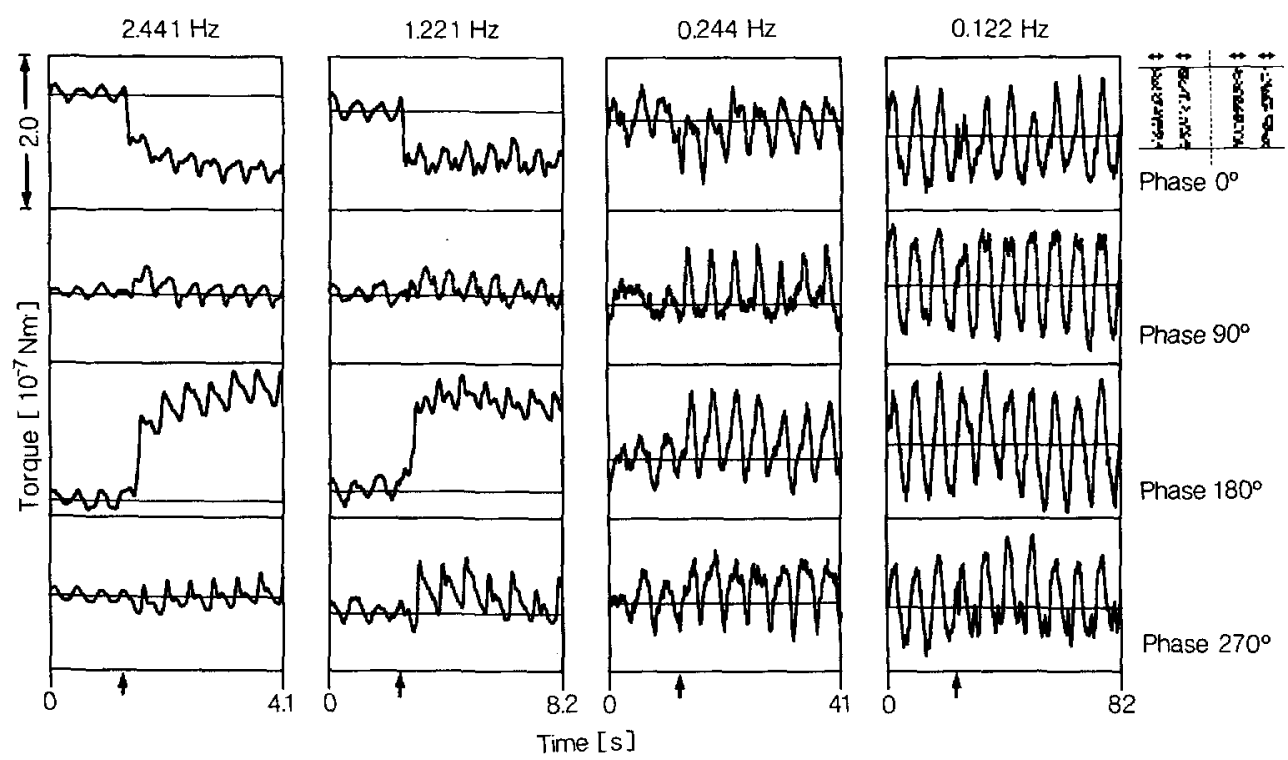

Fig. 4. Torque responses to the motion of two pairs of stripes. Both pairs oscillated horizontally (stimulus set-up (2); see inset) with different oscillation frequencies (indicated at the top of the different columns). The phase relation between the frontal and the lateral pair of stripes was varied (indicated at the right side of each row; the convention which defines the different phase relations is given in the text). At low oscillation frequencies, neither the time course of the responses, nor their mean amplitude is much affected by varying the phase relationship of relative motion. At high oscillation frequencies both features of the response profiles depend in a characteristic way on the phase relationship. The data are averages of 10 different flies each stimulated 10 times by the same stimulation program

fly still tries to follow the frontal stripes with yaw torque oscillations of relatively large amplitudes. Hence, under these conditions the fly does not respond to relative motion in any obvious way. In contrast, at high oscillation frequencies, the responses differ considerably both with respect to their mean amplitude and their time course from those to synchronous motion of the frontal stripes alone. Since the phase relations of $0^{\circ}$ and $180^{\circ}$, on the one hand, and of $90^{\circ}$ and $270^{\circ}$, on the other hand, are mirror-symmetrical with respect to stimulation of the left and right eye, the corresponding responses are also mirror-images of each other. During relative motion with a phase shift of $0^{\circ}$ and $180^{\circ}$ the mean torque responses are negative and positive, respectively. This implies that the fly tries to turn towards its left or right side. The time course of the response is no longer sinusoidally modulated but has a particular time course. During relative motion with a phase shift of $90^{\circ}$ and $270^{\circ}$ the mean responses are approximately zero. Nevertheless, the fly responds to these conditions of relative motion in a characteristic way, since the time course of the responses changes conspiciously as compared with synchronous oscillation of the frontal stripes. Small response peaks towards the right and left alternate with brief more or less pronounced plateau phases in between. These data show thatat high oscillation frequencies the responses are characteristic fingerprints of the different phase relations of relative motion. This is not true at low frequencies where no obvious responses to relative motion are visible. Based on the earlier conclusion that the yaw torque responses at low and high frequencies are mainly controlled by the LF- and SF-system (Egelhaaf 1987), these findings suggest that both control systems respond in a characteristic, but quite different way to relative motion. Hence, their spatial input organization may differ considerably. This information will be used in the next section to derive cellular models of their input circuitry.

\section{Cellular Models of the Large-Field and Small-Field System}

In a previous behavioral study (Reichardt et al. 1983) model circuits were proposed which could account for the specific features of the torque responses to coherent large-field motion and relative motion. All these models were based on the assumption that yaw torque as induced under these stimulus conditions is mediated by only a single control system. Two features of the responses were particularly critical in designing these models (Reichardt et al. 1983): (i) The characteristic dependence of the mean reaction on the angular horizontal extent of the stimulus pattern was found, under the conditions used in the experiments, to be essentially independent of pattern size. (ii) The characteristic time course of the responses to relative motion was found to represent a good fingerprint of 
the different phase relations between figure and ground motion which significantly constrained the possible model circuits. These model circuits were formulated and graphically represented in a way lending themselves well to an interpretation in cellular terms. Essentially three model versions were proposed. All of them assume two types of large-field elements on each side of the brain, a pool cell and an output cell. Both summate the output of the horizontally aligned local movement detectors. The pool cells on both sides of the visual system were proposed to be coupled. The output cells of the circuit are excited by front-to-back motion and are inhibited by back-to-front motion, the pool cells were originally concluded not to be directionally selective. The three model versions differ in the way the pool cells affect the spatial integration properties of the output cell. (i) In the forward model the individual movement detector channels are inhibited via shunting inhibition prior to summation by the output cell (Reichardt et al. 1983). (ii) In the recurrent model shunting inhibition operates on the individual movement detector channels prior to their summation by the pool cells (Reichardt et al. 1983). (iii) In the postsynaptic shunting inhibition model the pool cells do not inhibit the local movement detectors but directly the output cell of the network (Egelhaaf 1985a). In all of these models, the signals of the heterolateral output cells are subtracted from each other and then control the motor output of the circuit via a direct pathway and a lowpass filtered version of this signal. All these circuits lead to qualitatively the same responses and, therefore, could not be distinguished experimentally. This simple scheme had to be altered when it was realized in further behavioral and electrophysiological experiments that the yaw torque is jointly mediated by two control systems, the LS- and the SF-system, with different spatial integration properties (Egelhaaf $1985 \mathrm{a}, \mathrm{c})$. Instead of only one model circuit, two partly separate ones were now proposed (Egelhaaf 1985c). All these conclusions, however, were afflicted with one important limitation: It was not possible at the behavioral level to derive independently the specific features of the two parallel control systems and to assess their relative contribution to the behavioral responses. This was only possible, at least to some extent, when it was shown that both control systems have different dynamical properties, with the LF- and the SF-system controlling yaw torque mainly at low and high oscillation frequencies, respectively (Egelhaaf 1987). On this basis, it was necessary to reconsider the circuits responsible for the different spatial integration properties of both control systems.

The input to both circuits representing the LF- and the SF-system, respectively, is provided by local movement detectors. These are organized in two- dimensional retinotopic arrays which cover the entire visual field of both eyes. The computations performed by the local movement detectors have been characterized in great detail in formal terms (for review see Reichardt 1987). In the time average, the movement detectors calculate a kind of spatiotemporal crosscorrelation of the time-dependent light intensity fluctuations at their two input channels. The response of an individual movement detector as a function of time, however, is more complex, since it is not constant even if the stimulus pattern passes the detector with a constant velocity. Instead, the response profiles are modulated in time in a characteristic way (Reichardt and Egelhaaf 1988; Egelhaaf et al. 1989a, b). For this reason, the movement detectors were included in the model simulations. This is in contrast to our earlier papers on this issue (Reichardt et al. 1983; Egelhaaf $1985 \mathrm{a}, \mathrm{c}$ ) where, for convenience, the output of the local movement detectors was assumed to be proportional to pattern velocity. In our computer simulations the individual movement detector channels were splitted up into two branches, one carrying only their positive $\left(v^{+}(t)\right)$, the other only their negative response components $\left(v^{-}(t)\right)$. It should be noted that the mechanisms underlying movement detection can be accounted for satisfactorily so far only by an algorithmic model. In contrast, the mechanisms responsible for the different spatial tuning of the LF- and SF-system can be interpreted in cellular terms. Hence, our overall circuit as proposed here is a kind of hybrid. However, attempts are being made towards unravelling also the cellular mechanisms responsible for the different computations of the algorithmic movement detector model (Egelhaaf and Borst 1989). So there is some hope that in the near future all the constituent elements of the circuits mediating yaw torque responses of the fly during coherent large-field and relative motion can be interpreted in cellular terms.

4.1 The LF-System. The specific properties of the LFsystem can be explained by various alternative models which differ principally with respect to the cellular mechanisms involved. Since at low oscillation frequencies the behavioral responses do not much depend on the phase relationship of the different moving pattern segments (Figs. 2 and 4), these responses do not provide any pronounced fingerprint which can be used to distinguish between the different models of the LFsystem. Hence, on the present experimental basis we cannot be very specific in this respect. Only two principally different possibilities shall be discussed here.

Of course, the properties of the LF-system can be explained in terms of all the earlier proposed model circuits (see above) (Reichardt et al. 1983; Egelhaaf 


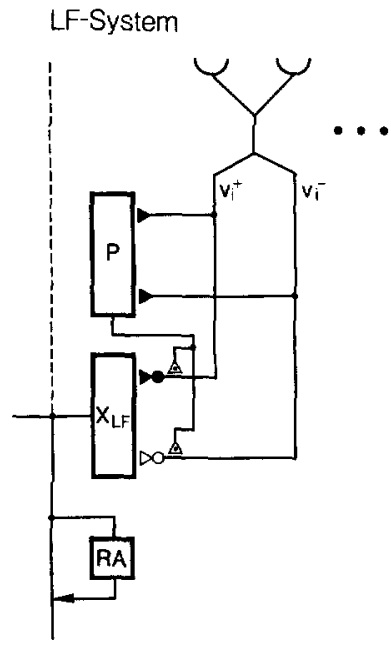

Fig. 5. Model of the LF-system. Input elements of the neural network are a retinotopic array of horizontally aligned local movement detectors (only a single detector is shown). These movement detectors synapse on two types of spatially integrating large-field cells. The output of the movement detectors segregates into two channels carrying only positive $\left(v_{i}^{+}\right)$and negative $\left(v_{i}^{-}\right)$ response components and independently controlling synapses. At the level of the "pool" $(P)$ cell both synapses are excitatory (i.e. the sign in the negative movement detector output channel is reversed). At the level of the output $\left(X_{\mathbf{L F}}\right)$ cell of the LF-system the positive movement detector channel and the negative channel contribute with an excitatory and an inhibitory synapse, i.e. with a positive and negative sign, respectively). The positive channels have a higher amplification than the negative ones $(1: 0.3)$. The $P$-cell is assumed to saturate and then shunts the movement detector channels near their output terminals via presynaptic inhibition. The synapses on the output cell operate with a nonlinear transmission characteristic. The final motor output is controlled by the output cells via a direct channel and a low-pass filtered version of it (RA). Since the networks of the LF-system on both sides of the brain operate independently, only the one on the right side is shown

1985a, c). The model version shown in Fig. 5 has essentially the same overall organization as the forward model already described above. It differs only in one important respect. The movement detector channels on one side of the brain are affected by only the ipsilateral pool cell of the model circuit. This leads to the following expression for the response of the right output cell of the LF-system

$$
R(t)=\sum_{i=1}^{N} \frac{\left[v_{i}^{+}(t)\right]^{n}-\left|v_{i}^{-}(t)\right|^{n}}{\left\{\beta+\left\{\sum_{i=1}^{N}\left[v_{i}^{+}(t)+\left|v_{i}^{-}(t)\right|\right]\right\}^{q}\right\}^{n}} .
$$

$v_{i}^{+}(t)$ and $v_{i}^{-}(t)$ represent the positive and negative response components of the different movement detectors; $\beta$ denotes the coefficient of shunting inhibition and $N$ the number of movement detector channels. The parameters $n$ and $q$ approximate the non-linear trans- mission characteritic of the movement detector synapses on the output cell and the saturation nonlinearity of the pool cell, respectively. They are constrained by the experimental results and were chosen in the model simulations appropriately $(n=1.25, q=0.5)$. These model parameters are not particularly critical as long as $n \cdot q<1$. Figures 7 and 8 show for the different stimulus conditions used in our experiments the corresponding model simulations of the LF-system. Indeed, these model simulations are similar to the torque responses obtained at low oscillation frequencies in that their time course does not much depend on the phase relationship between the different pattern elements which move relative to each other.

An alternative mechanism which may explain the behavioral responses equally well was suggested in an electrophysiological study on a particular class of motion-sensitive large-field cells in the third visual ganglion of the fly's brain (Hengstenberg 1982). This scheme essentially relies on intrinsic properties of neurons rather than on a circuit composed of different cells. It simply takes the inevitable saturation nonlinearities of nerve cells into account, supplemented by the assumption of a voltage-dependent potassium channel. Of course, the latter assumption is not unreasonable, since this type of membrane current is frequently encountered in different systems. Using the conventional equivalent circuit for the cellular membrane one can derive for the response of one output cell of the LF-system (see Appendix A) the following formal expression

$$
R(t)=\sum_{i=1}^{N} \frac{\left[v_{i}^{+}(t)\right]^{n}-\left|v_{i}^{-}(t)\right|^{n}}{\beta+\sum_{i=1}^{N}\left[v_{i}^{+}(t)\right]^{n}+\sum_{i=1}^{N}\left|v_{i}^{-}(t)\right|^{n}+c \cdot R(t)}
$$

with the variables and parameters having the same meaning as explained with respect to (1). It is interesting to note that under certain conditions this intracellular shunting model is equivalent to the recurrent model (Reichardt et al. 1983).

In conclusion, we do not have sufficiently specific experimental evidence at the behavioral level so far to distinguish between the different models for the LFsystem which may account for the functional properties of the torque responses at low oscillation frequencies. This situation is not much different if we take the response properties of the HS-cells, the likely cellular equivalent of the LF-system at the level of the third visual ganglion, into account (see Discussion).

4.2 The SF-System. With respect to the input organization of the small-field system we have much more specific information, since the behavioral responses have characteristic features under the different stimulus conditions (Figs. 2 and 4). Consequently, the po- 
ssible mechanisms which are responsible for these features can be constrained much more than in case of the LF-system. Most important, it is hardly possible to account for the specific spatial integration properties of the SF-system simply in terms of only intracellular mechanisms. The most obvious reason for this may be the fact that the activity of the small-field system is inhibited by motion in front of the contralateral eye, although there is no direct excitatory input from there. Therefore, only circuit models for the SF-system will be considered here. As in the earlier model versions (Reichardt et al. 1983; Egelhaaf 1985c) we have to assume some kind of pool cells in the input circuit of the network which spatially summate local movement detectors from both eyes and, thus, are most sensitive to large-field motion. These pool cells, however, cannot be assumed to be insensitive to the direction of motion. Instead, as a consequence of the finding that the figure is, on average, discriminated from its background when both oscillate with a high frequency and a phase shift of $180^{\circ}$ (Fig. 2), the pool cells are demanded to be, at least to some extent, directionally selective. Various schemes are possible to achieve this end. Here, we propose a version which appears to us plausible in terms of the neuronal hardware available in the fly's brain (see Discussion); it is similar to the model suggested earlier in an electrophysiological study on the cellular analogues of the SF-system (Egelhaaf $1985 \mathrm{c})$.

Two pool cells are proposed on each side of the brain, one $\left(P^{+}\right)$being activated by front-to-back motion and inhibited by back-to front motion and the other $\left(P^{-}\right)$being inhibited and excited by motion in the respective opposite directions. The inhibitory response components of the pool cells are weighted by a factor $T<1$ which approximates the common property of neurons that the reversal potential of inhibitory synapses is usually closer to the resting potential than of excitatory synapses. The responses of the pool cells are then given by the following formal expressions

$P^{+}(t)=\sum_{i=1}^{N}\left[v_{i}^{+}(t)+T \cdot v_{i}^{-}(t)\right]$,

$P^{-}(t)=-\sum_{i=1}^{N}\left[v_{i}^{-}(t)+T \cdot v_{i}^{+}(t)\right]$.

The pool cells from both sides of the brain are assumed to interact with the individual movement detector channels by shunting inhibition. Let us first consider the detector channels on the right side. Then, for convenience, $P_{\text {right }}^{+}$and $P_{\text {left }}^{-}$as well as $P_{\text {right }}^{-}$and $P_{\text {left }}^{+}$ may be combined to single expressions representing now binocular pool cells which show positive responses either when the stimulus panorama moves clockwise $\left(P_{\text {right }}^{c w}\right)$ or counter-clockwise $\left(P_{\text {right }}^{c c w}\right)$. The relative contribution of the ipsi- and contralateral input may differ, as is reflected by the factor $0<k<1$

$P_{\text {right }}^{c w}(t)=P_{\text {right }}^{+}(t)+k \cdot P_{\text {left }}^{-}(t)$,

$P_{\text {right }}^{c c w}(t)=P_{\text {right }}^{-}(t)+k \cdot P_{\text {left }}^{+}(t)$.

$P_{\text {right }}^{c w}$ and $P_{\text {right }}^{c c w}$ jointly interact with the individual movement detectors by shunting inhibition. For the shunting inhibition signal to be directionally selective, the relative contribution of both pool cells has to be weighted appropriately by a factor $k^{*}$ with $0<k^{*}<1$. With $k^{*}=1$ direction selectivity is lost. ( $P_{\mathrm{right}}^{c w}$ $\left.+k^{*} P_{\mathrm{right}}^{c c w}\right)$ and $\left(P_{\mathrm{right}}^{c c w}+k^{*} P_{\mathrm{righ}}^{c w}\right)$ now interact with those movement detectors on the right side which convey positive $\left(v^{+}\right)$and negative $\left(v^{-}\right)$signals, respectively ${ }^{1}$. The shunting inhibition input of the movement detector channels on the left side is organized mirrorsymmetrically. Moreover, the output of the different pool cells may saturate which is approximated by the exponent $q<1$ (with $q=0.5$ ) in our model simulations. After the shunting inhibition operation the responses of the detector channels carrying positive $\left(y^{+}\right)$and negative $\left(y^{-}\right)$signals, respectively, then read

$$
\begin{aligned}
& y_{i}^{+}(t)=\frac{v_{i}^{+}(t)}{\beta+\left[P_{\mathrm{right}}^{c w}(t)+k^{*} \cdot P_{\mathrm{right}}^{c c w}(t)\right]^{q}}, \\
& y_{i}^{-}(t)=\frac{v_{i}^{-}(t)}{\beta+\left[P_{\mathrm{right}}^{c c w}(t)+k^{*} \cdot P_{\mathrm{right}}^{c w}(t)\right]^{q}} .
\end{aligned}
$$

One further point should be mentioned. It is obvious that only positive values of the pool cell signals are admissible for the shunting operation. Negative values, therefore, are assumed not to lead to transmitter release and consequently are clipped. As in the earlier model, the individual movement detector channels are summated by the output cells of the network by excitatory and inhibitory synapses according to their sign. The synaptic transmission characteristic is nonlinear; this nonlinearity is represented by the exponent $n$ (with $n=3$ in our model simulations). Eventually, this leads to the following expressions for the responses of the right output cell

$$
R(t)=\sum_{i=1}^{N}\left[y_{i}^{+}(t)\right]^{n}-\left|y_{i}^{-}(t)\right|^{n}
$$

For the response of the left output cell one obtains the corresponding expression. The responses of the hetero-

\footnotetext{
${ }^{1}$ It should be noted that this model version is formally equivalent with another one where the influence of the pool cells on the movement detector channels as determined by the factor $k^{*}$ is avoided and $T$ is replaced by the redefined factor $T^{*}$ which now may also assume negative values. Both model versions are then related by the expression $T^{*}=\left(T-k^{*}\right) /\left(1-k^{*} T\right)$. Although this model version may be more parsimonious with respect to the necessary connections and parameters, it appears to us less plausible in neuronal terms
} 
lateral cells are finally subtracted from each other. As in the earlier models, the motor output of the network is controlled by the output cells via a direct pathway and a channel computing the running average of the output cell response.

This network model of the SF-system proved to be sufficient so far to account, together with the output of the LF-system, for the main characteristics of the yaw torque responses of the fly during stimulation with coherent large-field and relative motion at the different oscillation frequencies. The simulated responses of the SF-system model for the different stimulus conditions tested in our behavioral experiments are shown in Figs. 7 and 8; the different model parameters are specified in the figure legend. The weighted sum of the computed LF- and SF-system response is shown on the right hand side of Figs. 7 and 8 simulating the yaw torque response of the fly at high oscillation frequencies. Under these conditions the relative contribution of the SF-system dominates (for details see figure legend). As is obvious by comparing Figs. 7 and 8 with Figs. 2 and 4 the model simulations fit the experimental results quite satisfactorily with respect to the characteristic time course of the responses under the different stimulus conditions. It should be noted, that the best fits are obtained if the phase of the LFsystem is advanced by the phase of the SF-system by 0.11 cycle. This phase shift, however, is not critical, since even without it the experimental data are fitted sufficiently well.

\section{Discussion}

Two parallel bilaterally symmetrical control systems play a decisive role in mediating visually induced turning responses of the fly. These control systems are specifically tuned to different types of global motion patterns as may be induced on the eyes in different flight maneuvers. The large-field (LF-) system, on the one hand, is most sensitive to rotatory image displacements of the entire visual surround as occur during deviations of the fly from its course. Its output signals were concluded to be used to control corrective steering maneuvers. The small-field (SF-)system, on the other hand, is activated most strongly when a small object moves in front of the eye as well as during relative motion of objects and their background. The latter situation may occur when the fly passes a nearby object in front of a more distant textured background. The functional significance of the SF-system was concluded to involve the discrimination of objects from their background and the mediation of turns towards them. Both control systems do not only differ with respect to the global geometries of the retinal motion patterns they respond to most sensitively. Instead, they have also different dynamical response characteristics. The LF-system mediates optomotor turning responses mainly at low frequencies of retinal image displacements, whereas the SF-system is most active when small objects move with relatively high oscillation frequencies.

What computations are responsible for the different functional properties of the LF- and the SFsystem? This has been analyzed in various studies by both behavioral and electrophysiological techniques as well as by theoretical means (Poggio et al. 1981; Reichardt et al. 1983; Hausen 1984; Hausen and Wehrhahn 1983; Egelhaaf 1985a, 1987, 1989; Egelhaaf et al. 1988). The present study exploits the fortunate analytical situation that the LF- and the SF-system have different dynamical properties which allows to stimulate them relatively independently from each other (Egelhaaf 1987). The characteristic features of the responses induced at high and low oscillation frequencies were found to be to a large extent manifestations of the SF- and LF-system, respectively. In this way, it has been possible to investigate at the behavioral level, in some more detail than before, the mechanisms by which both control systems acquire their characteristic features and, in particular, their different spatial integration properties. These mechanisms have been derived here solely on the basis of behavioral experiments without reference to our extended knowledge on certain aspects of them as obtained at the neuronal level (Hausen 1982a, b; Reichardt et al. 1983; Egelhaaf 1985a, b, 1989; Egelhaaf et al. 1988; Hausen and Egelhaaf 1989). For this reason, we try to relate both levels of analysis and ask for the cellular identities of the different constituent elements of the model circuits proposed to represent these mechanisms.

The characteristic spatial integration properties of the SF- and the LF-system are mainly determined by (i) the local movement detectors which represent the input to the circuit, (ii) the large-field element which form the output of the circuit, and (iii) hypothetical pool elements which interact with either the individual movement detectors or directly with the output cells.

\section{The Retinotopic Movement Detector Input}

All mechanisms proposed to account for the specific properties of the LF- and SF-system have one feature in common: They receive direct excitatory and inhibitory input from retinotopic arrays of local movement detectors. From a functional point of view, the preferred direction of these movement detectors is along the horizontal axis of the eye. This can be concluded from experiments where the strength of the optomotor turning response was found to strongly 
depend on the direction of motion of a periodic grating, with the horizontal direction eliciting the largest response amplitudes (Götz 1968, 1983; Götz et al. 1979; Wehrhahn 1985, 1986; Zanker 1988). The same result was obtained for directionally selective motion-sensitive large-field neurons which are likely to be part of the LF-system (Hausen 1981, 1982b). It should be noted that these results do not imply that the local movement detectors responsible for this direction selectivity are necessarily anatomically aligned with the horizontal axis of the eye. Instead, there is good evidence that there are, in addition to horizontal detectors (Kirschfeld 1972; Riehle and Franceschini 1984; Schuling 1988), detectors which receive input from contiguous points of the hexagonal ommatidial lattice and, consequently, are inclined with respect to the horizontal axis of the eye (Buchner 1976; Zaagman et al. 1977; Götz and Buchner 1978; Schuling 1988). Nevertheless, since there are always pairs of them oriented mirror-symmetrically with respect to the eye's horizontal axis, the effective preferred direction of the summated activity of such pairs is again horizontal. These findings are important in the context of the present paper, since they corroborate the assumption made here that the output elements which directly mediate turning reactions about the fly's vertical axis are solely driven by horizontally oriented movement detectors.

The functional properties of these local movement detectors have been analyzed in great detail. A specific functional model specifying the different computations performed by a movement detector proved to be very successful so far to account for a large number of experimentally determined response properties under both steady-state and transient conditions. This model of the detector evaluates, on average, a kind of spatiotemporal cross-correlation of the light intensity fluctuations at two neighboring points in visual space (e.g. Reichardt 1961, 1987). An individual movement detector of this kind is not a pure velocity sensor that correctly indicates the direction and velocity of local motion. Instead, its response is strongly influenced by the textural properties of the moving pattern such as its spatial frequency content and contrast (Götz 1964, 1972; McCann and MacGinitie 1965; Eckert 1973; Buchner 1984; see also Reichardt and Guo 1986). Moreover, its response amplitude is not constant, even if the pattern passes the detector with a constant velocity, but is modulated in time depending on the local structure of the stimulus pattern (Reichardt and Egelhaaf 1988; Egelhaaf et al. 1989a, b). These peculiar properties of the local movement detector were the reason that in the present study they were included in our model considerations rather than making the approximation, as in our earlier papers on this subject
(Poggio et al. 1981; Reichardt et al. 1983; Egelhaaf $1985 \mathrm{a}, \mathrm{c})$, that the movement detector output is proportional to pattern velocity. These movement detectors can be assumed to represent the main retinotopic input of our model networks. To account for their positive and negative response components in cellular terms, the detector output is splitted up into two branches, carrying only positive and negative responses, respectively, and contacting the output cell via excitatory or inhibitory synapses. In this way, the output cells become directionally selective. Of course, other schemes are conceivable for the movement detectors to synapse on the output cells.

It should be noted, that we are aware of the fact that it is somewhat incongruent to include in this way the movement detectors into our model circuits. This is because the major computations performed by the movement detectors can be formulated only in mathematical terms and cannot be interpreted, so far, satisfactorily in cellular terms (see e.g. Egelhaaf and Borst 1989); the output channels of the detector, however, are interpreted as synapses. One reason for the difficulty to understand motion detection in cellular terms is the fact that the local motion detecting elements which are likely to represent the retinotopic local movement detectors in the fly's brain are relatively small and have been still elusive to an adequate systematic analysis. Nevertheless, there is cursory evidence from electrophysiological measurements on local neurons in the second visual ganglion, the medulla, which are sensitive to motion (Bishop et al. 1968; McCann and Dill 1969; Mimura 1971, 1972; DeVoe and Ockleford 1976; DeVoe 1980). Moreover, there is movement specific deoxyglucose labelling in this part of the brain (Buchner et al. 1984) which also suggests that at least certain aspects of local motion detection take place there.

\section{The Output Elements of the Circuit}

The core of both the LF- and the SF-system is formed by different large-field elements which receive their main input from the local movement detectors but differ considerably in their spatial integration properties. The response of the LF-system slightly increases with increasing width of the stimulus pattern, whereas the response of the SF-system initially increases, reaches an optimum and eventually decreases again. These differences are also reflected in the different time course of the responses to relative motion of different segments of the stimulus pattern (Figs. 2 and 4). Already in earlier studies it has been proposed that particular directionally selective motion-sensitive large-field elements in the third visual ganglion, the lobula plate, of the fly's brain may 
correspond to these model elements (for review, see Egelhaaf et al. 1988; Hausen and Egelhaaf 1989): While the HS-cells were assumed to represent the cellular analogue of the output element of the LFsystem (Hausen 1981, 1982a, b; Hausen and Wehrhahn 1983, 1989; Reichardt et al. 1983), the FD-cells are likely to represent the cellular equivalent of the SFsystem (Egelhaaf 1985b, c). This general interpretation could be corroborated in the present study in a more specific way. In particular, it could be shown that the time course of the behavioral responses at low oscillation frequencies and of the HS-cells is similar in that, under conditions of relative motion, it is not much influenced by the particular phase relations between figure and background (compare Fig. 2 with Fig. 26 in Reichardt et al. 1983). In contrast the behavioral responses at high oscillation frequencies and of the FD-cells both depend on the different stimulus conditions in a characteristic and similar way (compare Fig. 2 with Fig. 3 in Egelhaaf 1985c).

\section{Mechanisms Responsible for the Characteristic Spatial Tuning of the LF- and SF-System}

What mechanisms are responsible for the characteristic spatial integration properties of the LF- and SFsystem? In the present as well as in previous studies (Poggio et al. 1981; Reichardt et al. 1983; Egelhaaf 1985 c) various, partly related mechanisms have been proposed. Most of them rely on an inhibitory interaction of specific large-field elements, so-called pool cells, with either the individual local movement detectors or directly with the output cell of the network. These pool cells are believed to receive their input from local movement detectors. In the present study we could provide evidence that they are only postsynaptic to horizontally oriented detectors and are not affected by vertically oriented ones. This suggests that the processing of motion information by the systems preferentially responding to horizontal and vertical motion, respectively, remains separate as far as to the final motor output.

For the SF-system other mechanisms than this type of interaction with large-field pool cells are hardly conceivable. On the basis of the present behavioral experiments the properties of these pool cells can be considerably constrained. (i) Their receptive fields jointly cover the visual field of both eyes. (ii) They are expected to be directionally selective to motion. Various wiring schemes are possible which may realize these properties. Only one of them has been discussed in the present study. Two directionally selective pool cells are assumed which are sensitive to motion in front of both eyes. One of them is excited by ipsilateral motion from front-to-back as well as by contralateral

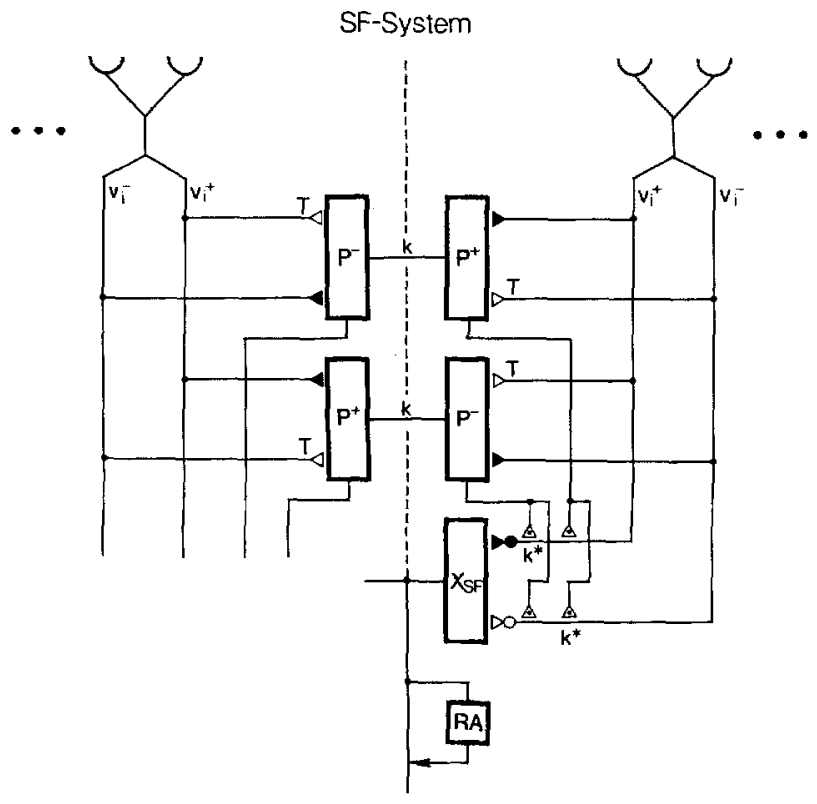

Fig. 6. Model of the input organization of the SF-system. It is similar to the one representing the LF-system as shown in Fig. 5 apart from some differences. There are two types of directionally selective binocular pool cells which receive positive and negative input from the movement detector channels (excitatory and inhibitory synapses are indicated by the filled and open synapses, respectively) in such a way that they are preferentially excited by clockwise or counterclockwise rotatory horizontal motion. The contribution of the contralateral side is weighted by the factor $0<k<1$. The contribution of the respective inhibitory input channels synapsing on the $P$-cells is weighted by the factor $T$, with $0<T<1$. These directionally selective pool cells interact with the individual movement detector output channels prior to their summation by the output cell in a way that can be inferred from the figure. The influence of one type of pool cell may be smaller than the other by the factor $k^{*}$ (with $0<k^{*}<1$ ). (For details see text)

motion from back-to-front; it is inhibited by motion in the respective opposite directions. In contrast, the other pool cell is assumed to have the opposite polarity. Of course, this scheme is only a functional concept and the specific realization of it shown in Fig. 6 is somewhat arbitrary. For instance, we cannot decide on the basis of our behavioral experiments, so far, whether the pool cells receive their motion-dependent input directly from retinotopically organized movement detectors or indirectly via other large-field neurons. Morover, the binocular pool cells are equivalent to two different uncoupled ones which jointly interact with the individual movement detector channels or directly with the putput cell of the network. These qualifications, however, are not relevant from a functional point of view.

Interestingly, there are cellular candidates, the $\mathrm{CH}$ and the H5-cells, in the third visual ganglion of the fly's brain which might represent these pool elements in the 

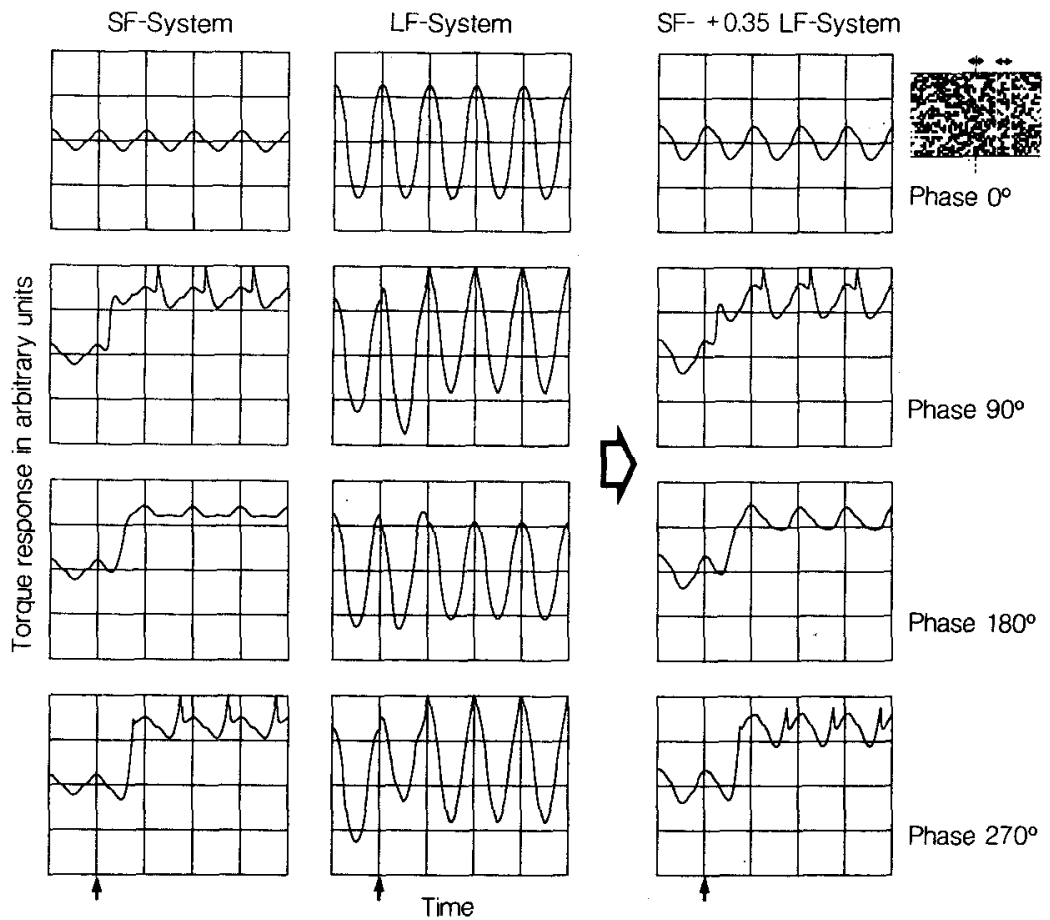

Fig. 7. Computer simulations of part of the experiments shown in Fig. 2. The simulations comprise: The movement detector level, the LF- and the SF-system, and the running average RA. Parameters of the simulations (see text) are: $\beta=0.6, k=0.7$, $T^{*}=-0.1$. The parameter $T^{*}$ has been explained in the footnote on page 336 . The additional contribution of the running average is determined by a factor 30 . The time-constant of the running average amounts to one temporal stimulus period. The results shown in the left and middle column are separate simulations of the SFand LF-system. The column on the right shows the combined activities of both control systems in order to simulate the corresponding experimentally determined behavioural responses at high oscillation frequencies (see left column in Fig. 2). After the contributions of the SF- and the LF-systems are (under phase $0^{\circ}$ conditions) normalized to one, the relative contribution of the LF-system to the right column amounts to $35 \%$ input circuitry of the FD-cells as has emanated mainly from studies of Hausen (Hausen in preparation; Hausen and Egelhaaf 1989). Both cells are centripetal elements projecting to the posterior part of the third visual ganglion from either the ipsi- or contralateral posterior optic foci of the ventral protocerebrum. Whereas the $\mathrm{CH}$-cells respond selectively to ipsilateral motion from front-to-back and contralateral motion from back-to-front (Hausen 1981; Eckert and Dvorak 1983), the H5-cell is excited by ipsilateral back-to-front and contralateral front-to-back motion (Hausen in preparation; Hausen and Egelhaaf 1989) just as proposed above for the pool cells. From their anatomy both cell types seem to be destined to reconvey information to the lobula plate and to interact in some way with the retinotopic array of input elements or directly with the dendritic tree of other large-field cells. It is therefore highly suggestive to speculate that these cells play a role in the input circuitry of the FD-cells.

The main reason for assuming directionally selective pool cells is our finding that, at high oscillation frequencies, the fly is able to discriminate the figure from its background if both oscillate in counterphase. This agrees with what was found under similar stimulus conditions in Drosophila (Bülthoff 1981), but is in contrast to earlier results obtained in Musca and Calliphora (Reichardt and Poggio 1979; Reichardt et al. 1983). Here no significant responses towards the figure could be detected under these stimulus conditions. This finding was the reason for postulating in the earlier study bidirectional pool cells that showed the same responses to motion in opposite directions. This discrepancy between the earlier and our present results cannot be resolved so far. Two observations, however, may be important to assess possible reasons. (i) The conclusions drawn in both studies are based on averaged responses. Careful analysis of the resonse traces obtained from individual flies reveals a considerable variability in this respect in both samples of flies. (ii) After switching the relative phase between figure and ground from $\phi=0^{\circ}$ to $\phi=180^{\circ}$, the mean response level is in some flies first shifted towards the position of the figure and then may decline again towards the zero line. Although the interpretations concerning the directional selectivities of the proposed pool cells as derived in the present and the earlier study (Reichardt et al. 1983) appear, at first sight, to be principally different, all experimental results can be explained by the same pair of directionally selective pool cells. By simply varying their relative contribution to the inhibition of the movement detectors, the divergent results found during counterphase oscillation of figure and ground can be easily accounted for. The extreme case of both directionally selective pool cells having the same share would be indistinguishable from the action of only a single bidirectional pool cell as proposed in the earlier study (Reichardt et al. 1983). It is interesting to note that there are FD-cells with functional properties corresponding to both types of pool organization. The activity of the right FD1-cell, for instance, is reduced by clockwise large-field motion in front of 

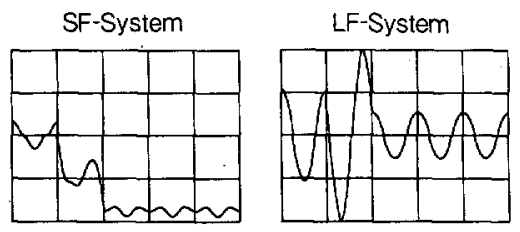

SF- +0.35 LF-System
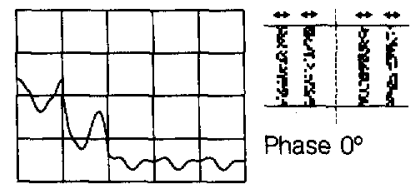

Phase $0^{\circ}$
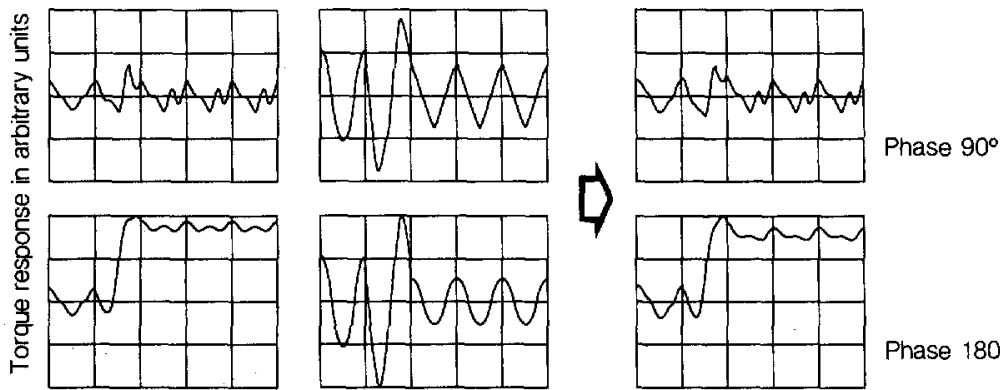

Phase $180^{\circ}$
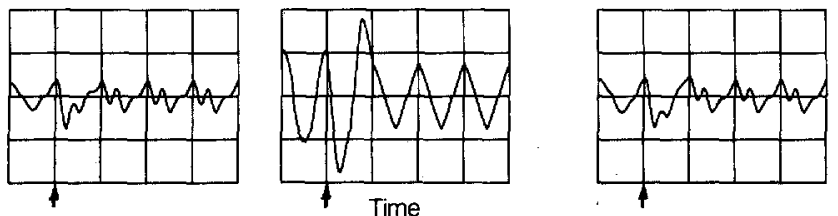

Phase $270^{\circ}$
Fig. 8. Computer simulations of part of the experiments shown in Fig. 4. The model parameters are as specified in the legend of Fig. 7 either eye. The pool cell in its input circuit, thus, appears to be directionally selective. In contrast, the activity of the FD4-cell decreases during large-field motion in both directions (Egelhaaf 1985b). This is consistent with the assumption of a single bidirectional pool cell or two directionally selective ones with opposite polarity (Egelhaaf 1985c). This suggests that, depending on which type of FD-cell predominates in its influcence on the motor output, figure-ground segregation at a phase shift of $180^{\circ}$ between figure and ground may be or may be not accomplished. How strong the different FD-cells affect the final motor output may depend, at least partly, on their different sensitivity for the different stimulus parameters. For instance, the spatial sensitivity distributions of the different FD-cells differ to some extent (Egelhaaf 1985b). There might be, however, other visual as well as non-visual determinants which may lead to a differential weighting of the FD-cell responses before they converge in some way in the motor control centers.

So far we do not have any direct evidence for the existence of pool cells in the input circuitry of the LFsystem. Nevertheless, as already done in earlier studies (Reichardt et al. 1983; Egelhaaf 1985a) the characteristic properties of the LF-system and its likely cellular analogue, the HS-cells, can be readily accounted for on the basis of this type of model circuit. Only the parameter characterizing the nonlinear transmission characteristic between the local movement detectors and the model HS-cell has to be chosen appropriately. Moreover, the pool cells have to be monocular in this case, since the activity of HS-cells is not reduced by motion in front of the contralateral eye. Further electrophysiological evidence demonstrates that the hypothetical pool elements in the input circuit of the HS-cells do not cover the entire vertical extent of the visual field but just the receptive field of the individual HS-cells (Egelhaaf, unpublished). Their receptive fields reach only over approximately $1 / 3$ of the vertical extent of the eye (Hausen 1982a, b). So far, we do not have any plausible candidate from anatomical or electrophysiological studies which meets all these requirements and may act as pool cell in the input circuit of the LF-system or the HS-cells.

A completely different possibility to account for the spatial integration properties of the HS-cells traces back to a proposal of Hengstenberg (1982). This intracellular shunting model essentially relies on intrinsic properties of neurons rather than on a circuit composed of different cells. This model simply takes the inevitable saturation non-linearities of nerve cells into account, supplemented by the assumption of a voltage-dependent potassium channel which reduces depolarizing membrane potential changes (see Appendix). The appeal of this model lies in the fact that it is rather parsimonious with respect to assumptions which go beyond mechanisms which are present in nerve cells anyway. Nevertheless, the available information on the properties of the LF-system are not specific enough, so far, to distinguish between the different model versions in either behavioral or electrophysiological experiments.

Although the models can predict the responses of the HS-cells and the behavioral responses during 
stimulation at low oscillation frequencies to both coherent large-field motion and relative motion sufficiently well, it is in two respects a very simplified representation of the neuronal network as it is actually implemented in the fly's brain. It does not take into account an experimentally established input in part of the HS-cells which is selectively activated by motion from the back to the front in the contralateral visual field. This input which is mediated by another largefield neuron of the contralateral lobula plate enhances the sensitivity of the HS-cells to rotatory large-field motion (Hausen 1982a, b). It could not be resolved on the basis of our behavioral measurements.

\section{Are these Mechanisms for the Evaluation of Different Global Retinal Motion Patterns of Significance Beyond the Fly?}

The evaluation of coherent large-field motion and relative motion between objects and their background appears to be of general relevance in visual information processing. Therefore, it is not much surprising that similar basic processing steps as found in the fly visual system seem also to play a role in other animals including man.

The best experimental evidence in favor of equivalent principles of information processing in different biological systems is available for the mechanism of local motion detection. Various psychophysical studies on man (e.g. van Doorn and Koenderink 1982a, b; van Santen and Sperling 1984; Wilson 1985; Baker and Braddick 1985) and electrophysiological studies on motion sensitive neurons in the cortex of cats (Emerson et al. 1987; Baker and Cynader 1988) indicate that essentially the same computations underly motion detection in these systems as originally derived in the insect visual system (for review see Borst and Egelhaaf 1989).

It is much more difficult to gain a coherent view with respect to the mechanisms operating on the output signals of the two-dimensional arrays of movement detectors. The available experimental evidence, however, indicates the existence of separate systems specifically tuned to the extraction of large-field motion as well as small-field and relative motion in different species. Both types of motion patterns, for instance, are represented at the single cell level. Cells most sensitively responding to coherent large-field motion have been found in different insect species (for review, see Wehner 1981). Since at least part of these neurons get input from both eyes and are activated by rotations of the entire visual surround, they are suggested, in analogy to the HS-cells and the LFsystem in flies, to play a role in mediating compensatory optomotor responses. In vertebrates, the
Accessory Optic System contains mainly neurons which are responsive to large-field motion. This system, therefore, has been concluded to be involved in the control of compensatory eye and head movements (for review, see Simpson 1984). There is much less uniformity among the different neurons selectively tuned to retinal small-field motion in both insects (e.g. Collett 1971; Collett and King 1975; Olberg 1981, 1986; Palka 1972; Rowell et al. 1977) and vertebrates (e.g. Frost et al. 1981; von Grünau and Frost 1983; Allman et al. 1985; Tanaka et al. 1986). This difference might be due to different behavioral contexts in which small objects moving relative to the eyes have to be detected and our, apart from few examples, complete lack of knowledge in this respect. One example, however, may suffice to illustrate that at least part of these cells may be involved in related tasks as the SFsystem and the FD-cells in the fly visual system. Neurons with properties reminiscent of the FD-cells have recently been found in the middle temporal region (area MT) of the monkey cortex (e.g. Allman et al. 1985; Tanaka et al. 1986). Since lesions of this area lead to deficits in the tracking of small moving objects, these cells have been proposed to play a role in the control of eye movements (Newsome et al. 1985; Newsome and Wurtz 1988) and thus might serve related purposes to the FD-cells.

Of course, by these similarities alone it cannot be assessed whether also similar mechanisms are responsible for these representations of different retinal motion patterns. Unfortunately, there is only sparse evidence in this respect. In systems extracting relative motion it seems to be a quite common feature that different parts of the visual field with diverging direction selectivities interact in a complex way. For instance, cells in both area MT in the monkey (e.g. Allman et al. 1985) and the optic tectum of the pigeon (Frost et al. 1981; Frost and Nakayama 1983) are influenced from beyond their classical excitatory receptive field. These areas can cover considerable parts of the visual field. Nevertheless, there is not sufficient information in these systems to assess in what way the different parts of the visual field interact to shape the response properties of these cells. To our knowledge, there is only one series of studies which propose a formal model to account for the specific integration properties of visual interneurons (Henn and Grüsser 1969; Grüsser 1971). Retinal ganglion cells in the cat were shown to have similar spatial integration properties as the HS-cells and the LF-system. Interestingly the model proposed to account for these features is very similar to our forward model (see above) in that it relies on "pool cells" (in this case assumed to be represented by the retinal horizontal cells) and presynaptic shunting inhibition. 
In conclusion, the fly visual system may be unique since only here it has been possible, so far, to gain a coherent view on the evaluation of different global retinal motion patterns. This has been possible at the different levels of analyzing complex biological information processing systems comprising both quantitative behavioral experiments as well as neurophysiological and theoretical investigations. The accessability of a system such as the visual system of the fly to a quantitative experimental analysis at both the behavioral and neuronal level underlines the importance of using comparatively simple systems to unravel the computations underlying different basic visual information processing tasks.

\section{Appendix: The Intracellular Shunting Model}

The intracellular shunting model was suggested, although not characterized in formal terms, in a study on directionally selective large-field neurons in the fly's third visual ganglion (Hengstenberg 1982). It relies, in contrast to the forward and the recurrent model, only on the biophysical properties of a single neuron, to achieve characteristic spatial integration properties. This neuron is assumed to integrate the signals of large retinotopic arrays of local movement detectors which converge on it by excitatory and inhibitory synapses. The equivalent electrical circuit of this model cell simplifies the geometrical relation of the neuron since it is assumed, as a first approximation, that the entire dendritic tree is isopotential. Since the transfer resistances between the different sites of synaptic input can then be neglected, the entire retinotopic input synapses can be lumped together to a common excitatory and inhibitory input channel, respectively. These two inputs control the conductances $g_{e}(t)$ and $g_{i}(t)$ of different ionic channels with equilibrium potential $E_{e}>E_{0}$ and $E_{i}<E_{0}$, respectively. $E_{0}$ is the resting potential of the cell which, for convenience, is set to zero. In addition to these synaptically controlled conductances, a voltage-dependent shunting conductance $g_{s h}$ with an equilibrium potential $E_{s h}$ is proposed which tends to suppress depolarizing membrane conductance changes. Voltagedependent potassium channels may be suitable for this purpose. The steady-state circuit equation can then be written as

$V(t)=\frac{E_{e} \cdot g_{e}(t)+E_{i} \cdot g_{i}(t)+E_{s h} \cdot g_{s h}(t)}{g_{e}(t)+g_{i}(t)+g_{s h}(t)+g_{0}}$.

This equation defines a non-linear relation between the different inputs of the cell and the cell's membrane potential $V(t) . g_{0}$ is the resting conductance of the cell. With appropriately chosen $g_{e}(t)$, $g_{i}(t)$, and $g_{s h}(t)$ the cell acquires specific spatial integration properties.

$g_{e}$ and $g_{i}$ are controlled by the positive and negative response components of the movement detector output signals $\left(v^{+}(t)\right)$ and $\left(v^{-}(t)\right)$. For simplicity, the following relations are assumed

$g_{e}(t)=\sum_{i=1}^{N}\left[v_{i}^{+}(t)\right]^{n}$

$g_{i}(t)=\sum_{i=1}^{N}\left|v_{i}^{-}(t)\right|^{n}$

$N$ represents the number of movement detector channels. The exponent $n$ approximates a nonlinear operation which trans- forms the presynaptic into the postsynaptic voltage. Moreover, $\mathrm{g}_{s h}$ is assumed to be proportional to the output of the cell $R(t)$ with $c$ being a constant:

$\mathrm{g}_{s h}(t)=c \cdot R(t)$.

If, for convenience, it is further assumed that $E_{s h}=E_{0}, E_{e}=1$, and $E_{i}=-1$, one obtains for the output of the model cell representing the core of the LF-system the following expression

$R(t)=\sum_{i=1}^{N} \frac{\left[v_{i}^{+}(t)\right]^{n}-\left|v_{i}^{-}(t)\right|^{n}}{\beta+\sum_{i=1}^{N}\left[v_{i}^{+}(t)\right]^{n}+\sum_{i=1}^{N}\left|v_{i}^{-}(t)\right|^{n}+c \cdot R(t)}$.

$\beta$ designates the resting conductance $g_{0}$. Of course, it might be more natural to assume that $\left|E_{i}\right|<\left|E_{e}\right|$, since the equilibrium potential of the inhibitory conductances is usually closer to the resting potential than of the excitatory conductances. It should be noted that for

$\beta+c \cdot R(t) \gg \sum\left[v_{i}^{+}(t)\right]^{n}+\sum\left|v_{i}^{-}(t)\right|^{n}$,

i.e. if the cell operates in a range of its input-output characteristic well below the level of saturation, (A3) becomes nearly equivalent to the equation characterizing the recurrent model (Reichardt et al. 1983).

Acknowledgements. We are indebted to Prof. K. G. Götz for critically reading the manuscript. In addition, we thank Prof. Götz for the graphics computer program which simulates the properties of a two-dimensional array of movement detectors as part of the entire simulation program. The simulations of the large-field and the small-field systems have been programmed by D. Opitz. We thank him for his delicate work. We also thank Mr. Leo Heimburger for preparing the figures and Mrs. Inge Geiss for secretarial help in typing different versions of the paper.

\section{References}

Allman J, Miezin F, McGuinness E (1985) Direction- and velocity-specific responses from beyond the classical receptive field in the middle temporal visual area (MT). Perception 14:105-126

Baker CL Jr, Braddick OJ (1985) Temporal properties of the short-range process in apparent motion. Perception 14:181-192

Baker CL Jr, Cynader MS (1988) Space-time separability of direction selectivity in cat striate cortex neurons. Vision Res 28:239-246

Bausenwein B, Wolf R, Heisenberg M (1986) Genetic dissection of optomotor behavior in Drosophila melanogaster. Studies on wild-type and the mutant optomotor-blind H31. J Neurogenet 3:87-109

Bishop LG, Keehn DG, McCann GD (1968) Motion detection by interneurons of optic lobes and brain of the flies Calliphora phaenicia and Musca domestica. J Neurophysiol 31:509-525

Borst A, Egelhaaf M (1989) Principles of visual motion detection. Trends Neurosci (in press)

Buchner E (1976) Elementary movement detectors in an insect visual system. Biol Cybern 24:85-101

Buchner E (1984) Behavioural analysis of spatial vision in insects. In: Ali MA (ed) Photoreception and vision in invertebrates. Plenum Press, New York London, pp 561-621 
Bülthoff $H$ (1981) Figure-ground discrimination in the visual system of Drosophila melanogaster. Biol Cybern 41:139-145

Collett TS (1971) Visual neurones for tracking moving targets. Nature 232:127-130

Collett TS, King AJ (1975) Vision during flight. In: Horridge GA (ed) The compound eye and vision of insects. Clarendon Press, Oxford, pp 437-466

DeVoe RD (1980) Movement sensitivities of cells in the fly's medulla. J Comp Physiol 138:93-119

DeVoe RD, Ockleford EM (1976) Intracellular responses from cells of the medulla of the fly, Calliphora erythrocephala. Biol Cybern 23:13-24

Doorn AJ van, Koenderink JJ (1982a) Temporal properties of the visual detectability of moving spatial white noise. Exp Brain Res 45:179-188

Doorn AJ van, Koenderink JJ (1982b) Spatial properties of the visual detectability of moving spatial white noise. Exp Brain Res 45:189-195

Eckert H (1973) Optomotorische Untersuchungen am visuellen System der Stubenfliege Musca domestica L. Kybernetik 14:1-23

Eckert H, Dvorak DR (1983) The centrifugal horizontal cells in the lobula plate of the blowfly, Phaenicia sericata. J Insect Physiol 29:547-560

Egelhaaf M (1985a) On the neuronal basis of figure-ground discrimination by relative motion in the visual system of the fly. I. Behavioural constraints imposed on the neuronal network and the role of the optomotor system. Biol Cybern 52:123-140

Egelhaaf M (1985b) On the neuronal basis of figure-ground discrimination by relative motion in the visual system of the fly. II. Figure-detection cells, a new class of visual interneurones. Biol Cybern 52:195-209

Egelhaaf M (1985c) On the neuronal basis of figure-ground discrimination by relative motion in the visual system of the fly. III. Possible input circuitries and behavioural significance of the FD-cells. Biol Cybern 52:267-280

Egelhaaf M (1987) Dynamic properties of two control systems underlying visually guided turning in house-flies. J Comp Physiol A 161:777-783

Egelhaaf M (1989) Visual afferences to flight steering muscles controlling optomotor responses of the fly. J Comp Physiol A (in press)

Egelhaaf M, Borst A (1989) On the role of GABAergic inhibitory synapses in motion detection of the fly. In: Elsner N, Singer W (eds) Dynamics and plasticity in neuronal systems. Thieme, Stuttgart New York, $\mathrm{p} 106$

Egelhaaf M, Hausen K, Reichardt W, Wehrhahn C (1988) Visual course control in flies relies on neuronal computation of object and background motion. Trends Neurosci 11:351-358

Egelhaaf M, Borst A, Reichardt W (1989a) The nonlinear mechanism of direction selectivity in the fly motion detection system. Naturwissenschaften 76:32-35

Egelhaaf M, Borst A, Reichardt W (1989b) Computational structure of a biological motion detection system as revealed by local detector analysis. J Opt Soc Am A (in press)

Emerson RC, Citron MC, Vaughn WJ, Klein SA (1987) Nonlinear directionally selective subunits in complex cells of cat striate cortex. J Neurophysiol 58:33-65

Fermi G, Reichardt W (1963) Optomotorische Reaktionen der Fliege Musca domestica. Abhängigkeit der Reaktion von der Wellenlänge, der Geschwindigkeit, dem Kontrast und der mittleren Leuchtdichte bewegter periodischer Muster. Kybernetik 2:15-28

Frost BJ, Nakayama K (1983) Single visual neurons code opposing motion independent of direction. Science 220:744-745

Frost BJ, Scilley PL, Wong SCP (1981) Moving background patterns reveal double-opponency of directionally specific pigeon tectal neurons. Exp Brain Res 43:173-185

Geiger G, Nässel DR (1982) Visual processing of moving single objects and wide-field patterns in flies: Behavioural analysis after laser-surgical removal of interneurons. Biol Cybern 44:141-149

Götz KG (1964) Optomotorische Untersuchung des visuellen Systems einiger Augenmutanten der Fruchtfliege Drosophila. Kybernetik 2:77-92

Götz KG (1968) Flight control in Drosophila by visual perception of motion. Kybernetik 4:199-208

Götz KG (1972) Principles of optomotor reactions in insects. Bibl Ophthalmol 82:251-259

Götz KG (1983) Bewegungssehen und Flugsteuerung bei der Fliege Drosophila. In: Nachtigall W (ed) BIONA report. Akademie der Wissenschaften und der Literatur zu Mainz and Fischer, Mainz Stuttgart New York, 21-34

Götz KG, Buchner E (1978) Evidence for one-way movement detection in the visual system of Drosophila. Biol Cybern 31:243-248

Götz KG, Hengstenberg B, Biesinger R (1979) Optomotor control of wing beat and body posture in Drosophila. Biol Cybern 35:101-112

Grünau M von, Frost BJ (1983) Double-opponent-process mechanism underlying RF-structure of directionally specific cells of cat lateral suprasylvian visual area. Exp Brain Res 49:84-92

Grüsser O-J (1971) A quantitative analysis of spatial summation of excitation and inhibition within the receptive field of retinal ganglion cells of cats. Vision Res [Suppl] 3:103-127

Hausen K (1981) Monocular and binocular computation of motion in the lobula plate of the fly. Verh Dtsch Zool Ges 74:49-70

Hausen K (1982a) Motion sensitive interneurons in the optomotor system of the fly. I. The horizontal cells: structure and signals. Biol Cybern 45:143-156

Hausen K (1982b) Motion sensitive interneurons in the optomotor system of the fly. II. The horizontal cells: receptive field organization and response characteristics. Biol Cybern 46:67-79

Hausen K (1984) The lobula-complex of the fly: Structure, function and significance in visual behaviour. In: Ali MA (ed) Photoreception and vision in invertebrates. Plenum Press, New York, pp 523-559

Hausen K, Egelhaaf M (1989) Neural mechanisms of visual course control in insects. In: Stavenga $\mathrm{D}$, Hardie $\mathrm{R}$ (eds) Facets of vision. Springer, Berlin Heidelberg New York, pp $391-424$

Hausen K, Wehrhahn C (1983) Microsurgical lesion of horizontal cells changes optomotor yaw responses in the blowfly Calliphora erythrocephala. Proc $\mathbf{R}$ Soc London $\mathbf{B}$ 219:211-216

Hausen K, Wehrhahn C (1989) Neural circuits mediating visual flight in flies. II. Separation of two control systems. Biol Cybern (submitted for publication)

Heisenberg M, Wolf R (1984) Vision in Drosophila. Springer, Berlin Heidelberg New York 
Hengstenberg R (1982) Common visual response properties of giant vertical cells in the lobula plate of the blowfly Calliphora. J Comp Physiol 149:179-193

Henn V, Grüsser O-J (1969) The summation of excitation in the receptive fields of movement-sensitive neurons of the frog's retina. Vision Res 9:57-69

Kirschfeld K (1972) The visual system of Musca: studies on optics, structure and function. In: Wehner R (ed) Information processing in the visual system of Arthropods. Springer, Berlin Heidelberg New York, pp 61-74

McCann GD, Dill JC (1969) Fundamental properties of intensity, form, and motion perception in the visual system of Calliphora phaenicia and Musca domestica. J Gen Physiol 53:385-413

McCann GD, MacGinitie GF (1965) Optomotor response studies of insect vision. Proc R Soc Lond B 163:369-401

Mimura K (1971) Movement discrimination by the visual system of flies. Z Vergl Physiol 73:105-138

Mimura K (1972) Neural mechanisms, subserving directional selectivity of movement in the optic lobe of the fly. J Comp Physiol 80:409-437

Newsome WT, Wurtz RH (1988) Probing visual cortical function with discrete chemical lesions. Trends Neurosci 11:394-400

Newsome WT, Wurtz RH, Dürsteler MR, Mikami A (1985) Deficits in visual motion processing following ibotenic acid lesions of the middle temporal visual area of the macaque monkey. J Neurosci 5:825-840

Olberg RM (1981) Object- and self-movement detectors in the ventral nerve cord of the dragonfly. J Comp Physiol 141:327-334

Olberg RM (1986) Identified target-selective visual interneurons descending from the dragonfly brain. J Comp Physiol 159:827-840

Palka J (1972) Moving movement detectors. Am Zoologist 12:497-505

Poggio T, Reichardt W, Hausen K (1981) A neuronal circuitry for relative movement discrimination by the visual system of the fly. Naturwissenschaften $68: 443-446$

Reichardt W (1961) Autocorrelation, a principle for the evaluation of sensory information by the central nervous system. In: Rosenblith WA (ed) Sensory communication. M.I.T. Press/Wiley, New York London, pp 303-317

Reichardt W (1987) Evaluation of optical motion information by movement detectors. J Comp Physiol A 161:533-547

Reichardt W, Egelhaaf M (1988) Properties of individual movement detectors as derived from behavioural experiments on the visual system of the fly. Biol Cybern 58:287-294

Reichardt W, Guo A (1986) Elementary pattern discrimination (behavioural experiments with the fly Musca domestica). Biol Cybern 53:285-306

Reichardt W, Poggio T (1979) Figure-ground discrimination by relative movement in the visual system of the fly. Part $\mathrm{I}$ : Experimental results. Biol Cybern 35:81-100

Reichardt W, Poggio T, Hausen K (1983) Figure-ground discrimination by relative movement in the visual system of the fly. Part II: Towards the neural circuitry. Biol Cybern 46 [Suppl]:1-30
Riehle A, Franceschini $N$ (1984) Motion detection in flies: parametric control over ON-OFF pathways. Exp Brain Res $54: 390-394$

Rowell CHF, O'Shea M, Williams JLD (1977) The neuronal basis of a sensory analyzer, the acridid movement detector system. IV. The preference for small field stimuli. J Exp Biol 68:157-185

Santen JPH van, Sperling G (1984) Temporal covariance model in human motion perception. J Opt Soc Am A 1:451-473

Schuling FH (1988) Processing of moving images in natural and artificial visual systems. Doctoral Dissertation, University of Groningen

Simpson J1 (1984) The accessory optic system. Ann Rev Neurosci 7:13-41

Tanaka K, Hikosaka K, Saito H, Yukie M, Fukada Y, Iwai E (1986) Analysis of local and wide-field movements in the superior temporal visual areas of the macaque monkey. J Neurosci 6:134-144

Virsik R, Reichardt W (1974) Tracking of moving objects by the fly Musca domestica. Naturwissenschaften 61:132-133

Virsik R, Reichardt W (1976) Detection and tracking of moving objects by the fly Musca domestica. Biol Cybern 23:83-98

Wehner R (1981) Spatial vision in insects. In: Autrum H (ed) Handbook of sensory physiology, vol VII/6C: Comparative physiology and evolution of vision in invertebrates. Springer, Berlin Heidelberg New York, pp 287-616

Wehrhahn C (1985) Visual guidance of flies during flight. In: Kerkut GA, Gilbert LI (eds) Comprehensive insect physiology, biochemistry and pharmacology. Pergamon Press, Oxford New York Toronto, pp 673-684

Wehrhahn C (1986) Motion sensitive yaw torque responses of the housefly Musca: A quantitative study. Biol Cybern 55:275-280

Wehrhahn C, Hausen K (1980) How is tracking and fixation accomplished in the nervous system of the fly? A behavioural analysis based on short tim stimulation. Biol Cybern 38:179-186

Wilson H (1985) A model for direction selectivity in threshold motion perception. Biol Cybern 51:213-222

Zaagman, WH, Mastebroek HAK, Buyse T, Kuiper JW (1977) Receptive field characteristics of a directionally selective movement detector in the visual system of the blowfly. J Comp Physiol 116:39-50

Zanker JM (1988) How does lateral abdomen deflection contribute to flight control of Drosophila melanogaster? J Comp Physiol A 162:581-588

Received: June 5, 1989

Prof. Werner Reichardt

Max-Planck-Institut für biologische Kybernetik

Spemannstrasse 38

D-7400 Tübingen

Federal Republic of Germany 